\title{
Is Outpatient Follow-Up of Epiploic Appendagitis with NSAIDs Alone and Noantibiotics Possible?
}

\author{
Mehmet İlhan*, Erçin Sönmez, Kaan Gök, Hakan Yanar, Recep Güloğlu, Kayhan Günay, \\ Cemalettin Ertekin \\ Department of General Surgery, Istanbul University School of Medicine, Istanbul, Turkey \\ Email: ${ }^{*}$ op.dr.mehmetilhan@gmail.com
}

Received 23 February 2014; revised 25 March 2014; accepted 2 April 2014

Copyright (C) 2014 by authors and Scientific Research Publishing Inc.

This work is licensed under the Creative Commons Attribution International License (CC BY).

http://creativecommons.org/licenses/by/4.0/

\section{Abstract}

Purpose: To assess the clinical course of patients diagnosed as having "epiploic appendagitis" who are given only NSAIDs without antibiotic treatment. Materials and Methods: Between December 2010 and November 2013, twelve patients were presented to the Emergency Surgery Department, Istanbul Medical Faculty, Istanbul University with abdominal pain and were diagnosed having "epiploic appendagitis". The diagnoses were made using the information gathered from patients' complaints, physical examination, biochemical results, and radiologic images (Abdominal X-Ray, Abdominal Ultrasonography [USG], and Contrast-Enhanced Computerized Tomography [CT]). We evaluated and recorded the patients' age distribution, sex difference, laboratory results, radiologic images, length of hospital stay, and control exams after discharge. Results: Twelve out of 35,574 $(\mathbf{0 . 0 3 3 \% )}$ patients who were presented to our Emergency Surgery Department with abdominal pain and were hospitalized as "epiploic appendagitis". Of these 12 patients, 7 were male (58.3\%), and 5 were female $(41.7 \%)$. The mean age of the patients was 43.5 (range: 22 to 60 ). The mean time passed between the beginning of the symptoms and presentation to the hospital was 1 day. The length of hospital stay was 1 to 2 days with a mean of 1.3 days. All patients were followed-up non-invasively under NSAID treatment without antibiotherapy. No complications were observed throughout the hospitalization period. The follow-up period of the patients was between 2 to 31 months with a mean of 16 months. Conclusion: Epiploic appendagitis is a self-limiting, benign disease, but differential diagnosis should be done carefully. Patients who are diagnosed with epiploic appendagitis may be followed-up with NSAIDs alone without hospitalization or antibiotherapy.

\section{Keywords}

Epiploic Appendagitis; Acute Abdomen; Acute Diverticulitis; Acute Appendicitis

\footnotetext{
*Corresponding author.
} 


\section{Introduction}

Epiploic appendices, which were first defined by Versailus, are lipid-rich peduncular structures covered by the colonic serosa, and their vascular supply is provided by two arteries and one vein [1]-[3]. They are prone to deformations as a result of their anatomic features. Epiploic appendices show symptoms mostly because of its torsion, ischemia or hemoragicinfarct [4] [5]. This acute abdomen presentation, which is defined as "primary epiploicappendagitis", occurs most commonly in the fourth decade of life [5]. Patients usually present to hospital with a one- or two-day complaint of abdominal pain. Usually, epiploic appendagitis is a self-limiting, benign disease. The diagnosis is made using abdominal X-ray, abdominal ultrasonography (USG), and contrast-enhanced computerized tomography (CT) [6] [7]. Today, the surgical approach has been replaced by medicinal treatment [7]. In this study, we evaluated the clinical course of patients diagnosed as having epiploic appendagitis who were given NSAIDs alone without any antibiotic treatment.

\section{Materials and Methods}

Patients with abominal pain who had presented to our emergency surgery department between December 2010 and November 2013, and were diagnosed as having epiploic appendagitis were enrolled into the study. We evaluated and recorded the patients' sex, age distributions, medical histories (known illnesses, previous operations, medications used) and the elapsed time between the beginning of the complaints and presentation to hospital were evaluated. After taking their medical history, routine abdominal examination of the patients were performed. Abdominal guarding and rebound tenderness positivity were noted. Also costovertebral angle tenderness was checked for possible renal colic.

The patients' blood and urine samples (CBC, chemistry panel, CRP levels and urinalysis) were analysed. Presence of erythrocytes, leucocytes and nitrite in urine were checked to rule out renal colic and urinary tract infection. Abdominal X-ray was performed on all patients. Either abdominal USG or CT was used depending on physical examination findings and the patient's physical features. Abdominal USG was performed by radiologists from the radiology department at different times. Definite diagnoses were reached by the contrast-enhanced (oral/iv/rectal) computerized tomography (CT). For contrast-enhanced abdominal CT imaging, 25 cc of contrast liquid (water soluble contrast solution) mixed with 500 cc of water was given to the patients to be consumed within an hour. An additional contrast-mixed solution was given via rectal/IV route by the radiology technician just before the images were taken.

All of the hospitalized patients received parenteral fluid treatment and proton pump inhibitors (Isolyte M + Pantoprazole $40 \mathrm{mg}$ iv $1 \times 1$ ) and oral nutrition was stopped until a definite diagnoses were made. After the diagnosis of epiploic appendagitis was reached, NSAIDs were added to the treatment, parenteral fluid was stopped, and a complete oral diet was resumed. Due to the NSAIDs in the treatment, oral proton pump inhibitors (PPI) were used for prophylactic gastric protection. None of the patients were given antibiotics before or after diagnosis. During the hospital stay and after discharge on the 2nd, 7th and 30th days, the patients were evaluated and physical examinations and blood analyses were performed.

\section{Results}

Twelve out of 35,574 (0.033\%) patients who presented to our emergency surgery department with abdominal pain and hospitalized were diagnosed as "epiploic appendagitis". Of these 12 patients, 7 were male (58.3\%), and 5 were female (41.7\%). The mean age of the patients was 43.5 (range: 22 to 60 years). The mean time elapsed between the beginning of the symptoms and presentation to the hospital was 25 hours (range: 8 to 42 hours). On the day of hospital presentation, physical examinations were made and recorded: 5 patients had tenderness in right lower quadrant, 5 in the left lower quadrant, and 2 in the right upper quadrant of the abdomen. There was abdominal guarding in 10 patients and rebound tenderness in 2 patients.

The findings were observed to disappear over time at consecutive daily physical examinations.

Nine of the patients had no history of abdominal operations. Each one of the remaining three had a history of surgery: one had an appendectomy, one had a histerectomy and the other had a caesarean section (C/S).On the day of hospital presentation, the mean number of leucocytes was recorded as $7.8 \times 10^{3} / \mu \mathrm{L}$ (range: 6.3 to $9.2 \times$ $\left.10^{3} / \mu \mathrm{L}\right)$. Throughout the follow-up period, these leucocytelevels stayed within the normal range $(4.8-10.8 \times$ $10^{3} / \mu \mathrm{L}$ ). The average CRP level was $43.7 \mathrm{mg} / \mathrm{L}$ (range: 2.8 to $164.5 \mathrm{mg} / \mathrm{L}$ ). Some of the patients had high levels of CRP at the beginning, but those values also decreased during the follow-up period and no deterioration of 
their clinical course was observed. Urinalyses of the patients were within normal limits.

Abdominal X-Rays only showed small and large intestinal gas shadows. Abdominal USG was performed on 8 patients. Four patients had no diagnostic features. Two had lesions in the left lower quadrant consistent with epiploic appendagitis and the remaining 2 had increasedechogenicity in the caecum-adjacent mesenteric fatty planes. In all cases, we observed diagnostic images using oral/iv/rectal contrast-enhanced computerized tomographies consistent with epiploic appendagitis. Inflamation and minimal reactional fluid collection was observed in adjacent mesenteric planes (Figure 1).

After the definite diagnosis was reached, NSAIDs were added to the treatment and parenteral PPIs were changed to oral forms. Parenteral fluid treatments of the patients who could tolerate enteral nutrition were stopped after a mean of 4 hours. After a mean of 1.3 days (range: 1 to 2 days) of hospital stay, the patients were discharged with a prescription of an oral NSAID and a PPI (Pantoprazole $40 \mathrm{mg}$ tb $1 \times 1$ ). All twelve patients were followed-up at 2, 7 and 30 days after their discharge and no problems were observed. No additional imaging was required. After detecting colonic mucosal thickening on the contrast-enhanced abdominal CTs of two patients, elective colonoscopies were performed on both and polyp excision was performed on one of them. The follow-up period for the patients was between 2 to 31 months with a mean of 16 months.

\section{Discussion}

Epiploic appendices are pedunculated lipid-rich structures covered by the colonic serosa which have an average width of 1 to $2 \mathrm{~cm}$ and a length of 0.5 to $5 \mathrm{~cm}$. Their function is similar to the omentum's defensive and protective mechanisms. Also, due to the lenfatic structures it contains, epiploic appendices play a role in the immune system. An average of 50 to 100 epiploic appendices is situated along the colonic wall, mostly under the serosa of transvers, descending and sigmoid colon especially. They can be more distinctive in the obese and people who lost weight rapidly [4] [5] [8].

The vascular supply of epiploic appendices is usually provided by 2 arteries and 1 vein within the pedunculated lipid tissue. Because of their pedunculated anatomy, limited vascular supply, and extensive capability of movement, they are prone to pathologies such as torsion, ischemia and hemoragic infarct. The clinic entity defined as "epiploic appendagitis" is seen mostly between the second and fifth decades of life, and has an incidence of 8.8 per million $(0.0008 \%)$. There is no gender difference in the incidence of epiploic appendagitis, although it is seen more commonly in obese people [5]. In our clinic, twelve of 35,574 cases $(0.033 \%)$ who presented to the hospital between the years of 2010 and 2013 with abdominal pain were diagnosed having "epiploic appendagitis". Five were female and seven were male patients, and their ages were between 22 and 64 years.

Patients with epiploic appendagitis present to hospital with abdominal pain mostly in the right, but sometimes the left lower quadrant of abdomen, which is present for a couple of days. There is usually no rebound tenderness. Fever and tachycardia are usually not observed. Leucocytosis is minimal, and sometimes high levels of
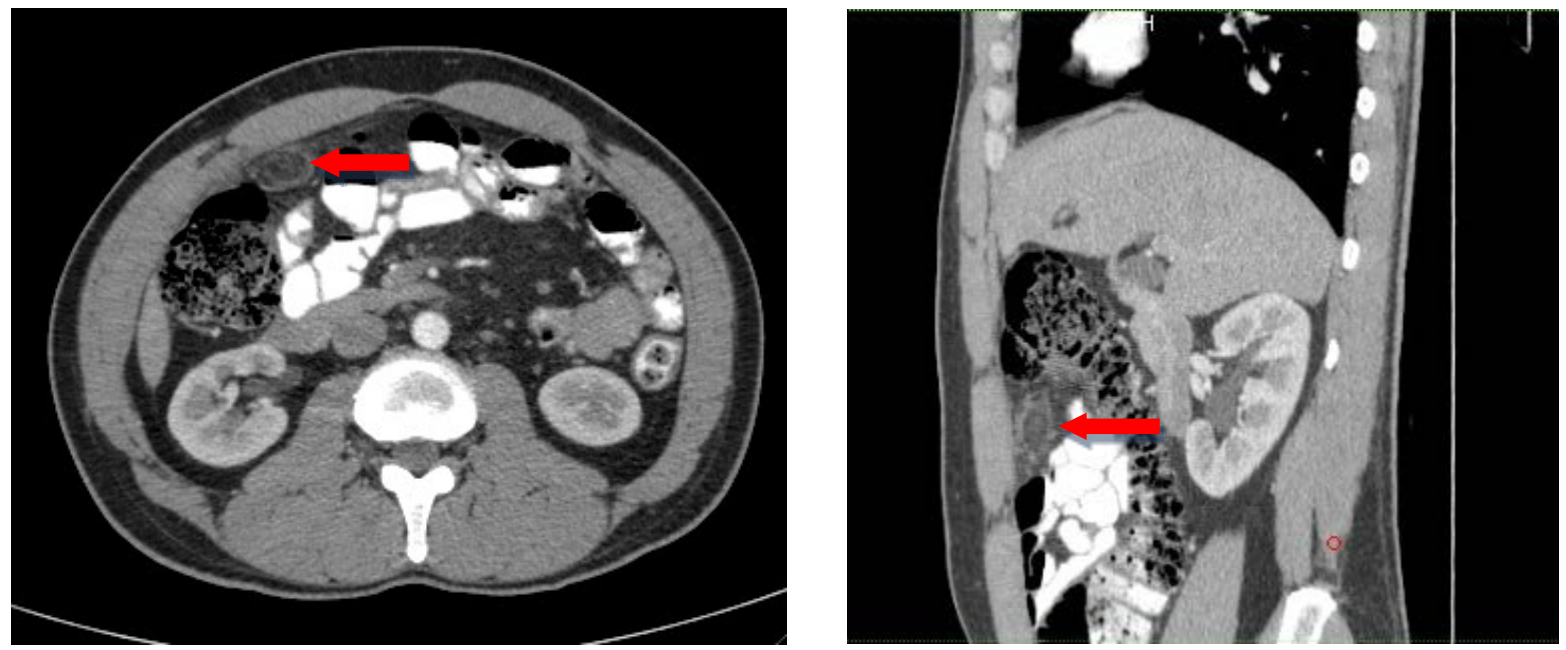

Figure 1. Transvers and longitudinal abdominal CT without contrast enhancement demonstrated the inflamed lesion (arrow) adjacent to the ascending colon and showed an oval area with a diameter of $2.5 \mathrm{~cm}$ surrounded by an edematous ring. 
CRP may be seen. Other than complicated cases, it is a self-limiting, benign disease [5]. In our physical examinations, 5 patients had tenderness in the right lower quadrant, 5 in the left lower quadrant, and 2 in the right upper quadrant. The leucocyte levels were within the normal limits $\left(4.8-10.8 \times 10^{3} / \mu \mathrm{L}\right)$.

The mean CRP level was $43.7 \mathrm{mg} / \mathrm{L}$ (range: 2.8 to $164.5 \mathrm{mg} / \mathrm{L}$ ). CRP levels that were above the upper limits decreased dramatically during the follow-up period. Abdominal ultrasonography may be a suitable option in the diagnosis-making procedure because it is a cost-effective, and non-invazive procedure. On sonography, epiploicappendagitis shows itself as a non-compressible, hypoechoic solid lesion with clear borders, surrounded by a hypoechoic ring. However, the gold standard imaging technique for epiploicappendagitis is contrast-enhanced computerized tomography (CT). Normally, epiploicappendices are not seen on CT. In the presence of inflammation, they are seen aspedunculated structures. Also, abdominal magnetic resonance imaging (MRI) may be useful to assess the extent of inflammation [6]. In our patients, no pathologic features were seen onabdominal Xrays. Four patients had diagnostic features on abdominal ultrasonography (USG) and definite diagnoses were reached using contrast-enhanced computerized tomography (CT).

Due to this, oral/iv/rectal contrast-enhanced abdominal CT can be accepted as the goldstandard approach to reach diagnosis, having a near 100\% sensitivity and true positivity rate (Figure 2).

Primary epiploic appendagitis used to be treated surgically, however patient follow-ups and new studies showed the surgical approach to be unnecessary and that conservative medical follow-up was an appropriate treatment model. It is understood that the disease is self-limiting and has a benign clinical course [7]. One of the most important points is to make the differential diagnosis correctly. To achieve this, the features and clinical course of the disease should be fully understood. Acute appendicitis, acute cholecystitis, acute diverticulitis, mesenteric panniculitis, omental infarct, and neoplasms are among the most common pathologies that should be considered in a differential diagnosis [8]. In complicated, nonremissingor relapsing cases such as pericolic abscesses, tight attachments in the abdomen and resulting possible brid ileus, and intusseption, diagnostic approaches may be an option. [9] In our series, no complications were observed.

Based on the pathophysiology of anti-inflammation, many authors declare that the use of NSAIDs alone (especially ibuprophen) without any antibiotics is adequate. There are other studies addressing follow-up with NSIADs treatment alone in other clinics. Some authors think that outpatient follow-up is appropriate and patients do not require hospitalization [7]. The debate about the use of antibiotherapy and NSAIDs still continues.

In our study, after reaching the definite diagnosis, the parenteral treatment was stopped and full oral diet was given to all of our patients. Non-steroidal antiinflammatory drugs and PPI were given as the medical treatment. The hospitalization period of the patients was between 1 to 2 days with a mean of 1.3 days. The patients were asked to come to follow-up at 2, 7 and 30 days after discharge. None of the patients had problems, and therefore no additional radiological evaluation was required. In conclusion, primary epiploic appendagitis is a benign disease presenting as acute abdomen, and can be managed by the use of NSAIDs alone without hospitalization or antibiotherapy. Therefore, it is vital to make the differential diagnosis correctly. By doing so, a possible invasive
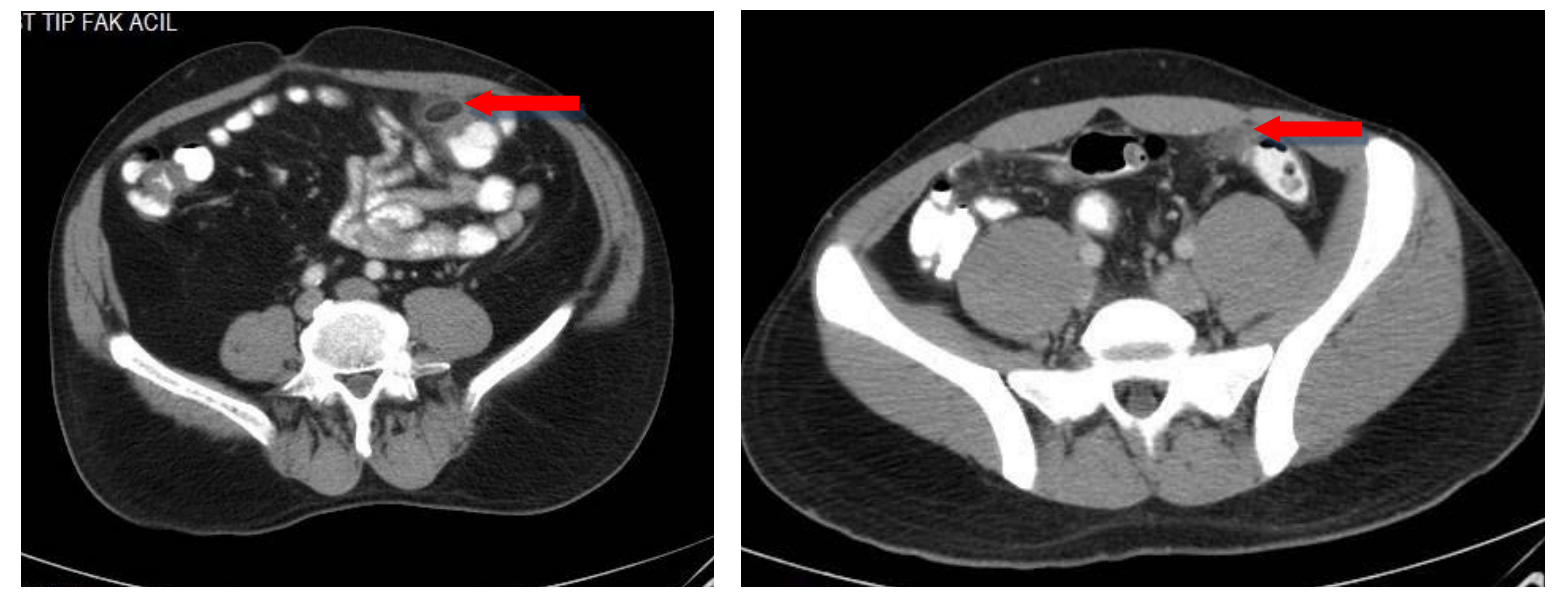

Figure 2. On CT scan, there is inflamed appendix epiploica, appearing as a lipiddensitylesion surrounded by hyperdens rim, and peripheric serozal enflammation on sigmoid and descending colonic locations). 
approach can be avoided, and lower morbidity and mortality rates can be achieved. Studies with a greater number of subjects may lead to more meaningful results.

\section{References}

[1] Garg, P.K., Verma, R., Jain, B.K. and Aggarwal, S. (2013) Epiploic Appendagitis. An Uncommon Cause of Acute Abdomen, 12, 257-258.

[2] Malay, Y.B., Santiago, M.-J., Rosado-de-Christenson, M.L., Kenneth, R.W., Christopher, M.W. and Jeffrey, R.K. (2013) Case Report, Imaging Manifestations of Mediastinal Fat Necrosis. Case Reports in Radiology, 2013, 323579.

[3] Savage, L., Gosling, J., Suliman, I., Klein, M.B.M.J. (2013) Case Rep. Epiploic Appendagitis with Acute Appendicitis. http://dx.doi.org/10.1136/bcr-2013-010333

[4] Wolfgang, J.S., Robert, K., Erwin, T., Manfred, T., Rainer, W.L. and Sandra, J.W.-L. (2011) Insights into Epiploic Appendagitis. Nature Reviews Gastroenterology and Hepatology, 8, 45-49. http://dx.doi.org/10.1038/nrgastro.2010.189

[5] Choi, Y.U., Choi, P.W., Park, Y.H., Kim, J.I., Heo, T.G., Park, J.H., Lee, M.S., Kim, C.N., Chang, S.H. and Seo, J.W. (2011) Clinical Characteristics of Primary Epiploic Appendagitis. Journal of Korean Social Coloproctology, 27, 114121. http://dx.doi.org/10.3393/jksc.2011.27.3.114

[6] Oztunali, C. and Kara, T. (2013) Ultrason. Radiologic Findings of Epiploic Appendagitis, 15, 71-72.

[7] Rashid, A., Nazir, S., Hakim, S.Y. and Chalkoo, M.A. (2012) German Medical Science. Epiploic Appendagitis of Caecum: A Diagnostic Dilemma.

[8] Hwang, J.A., Kim, S.M., Song, H.J., Lee, Y.M., Moon, K.M., Moon, C.G., Koo, H.S., Song, K.H., Kim, Y.S., Lee, T.H., Huh, K.C., Choi, Y.W., Kang, Y.W. and Chung, W.S. (2013) Differential Diagnosis of Left-Sided Abdominal Pain: Primary Epiploic Appendagitis vs Colonicdiverticulitis. World Journal of Gastroenterology, 19, 6842-6848. http://dx.doi.org/10.3748/wjg.v19.i40.6842

[9] Uehara, R., Isomoto, H., Yamaguchi, N., et al. (2011) Epiploic Appendagitis in a 27-Year-Old Man. Medical Science Monitor, 17, CS113-CS115. http://dx.doi.org/10.12659/MSM.881968 